\title{
Mild Bradykinesia Due to an Injury of Corticofugal-Tract from Secondary Motor Area in a Patient with Traumatic Brain Injury
}

Han Do Lee', Jeong Pyo Seo²

'Department of Physical Therapy, College of Natural Science, Ulsan College University, Ulsan, Republic of Korea; ${ }^{2}$ Department of Physical Therapy, College of Health and Welfare Sciences, Dankook University, Cheonan, Republic of Korea

Objectives: We report on a patient who showed mild bradykinesia due to injury of the corticofugal tract (CFT) from the secondary motor area following direct head trauma, which was demonstrated on diffusion tensor tractography (DTT).

Case summary: A 58-year-old male patient underwent conservative management for subarachnoid hemorrhages caused by direct head trauma resulting from a fall from six-meter height at the department of neurosurgery of a local hospital. His Glasgow Coma Scale score was 3. He developed mildly slow movements following the head trauma and visited the rehabilitation department of a university hospital at ten weeks after the fall. The patient exhibited mild bradykinesia during walking and arm movements with mild weakness in all four extremities $\left(\mathrm{G} / \mathrm{G}^{-}\right)$.

Results: On ten-week DT, narrowing of the right CF from the supplementary motor area (SMA-CFT), and partial tearing of the left SMA-CF, left CFTs from the dorsal premotor cortex (dPMC-CFT) and both corticospinal tracts (CSTs) at the subcortical white matter were observed.

Conclusion: This case demonstrated abnormalities in both CSTs (partial tearing at the subcortical white matter and narrowing), both SMA-CFTs (narrowing and partial tearing) and left dPMC-CF. We believe our findings suggest the necessity of assessment of the CFTs from the secondary motor area for patients with unexplained bradykinesia following direct head trauma.

Keywords: Corticofugal tract, Diffusion tensor tractography, Bradykinesia, Direct head trauma

\section{INTRODUCTION}

The corticofugal tracts (CFTs) come from the premotor or non-primary motor or premotor cortices are classified according to the CFT from the dorsal and ventral premotor cortex (dvPMC), and supplementary motor area (SMA). ${ }^{1}$ Injury of the CFTs is usually accompanied by limb kinetic apraxia, because the CFTs from the secondary motor area are involved in motor planning. ${ }^{2}$ Bradykinesia is a typical mild symptom of limb kinetic apraxia. ${ }^{2}$ However, the relation between CFT and bradykinesia has not been elucidated.

The introduction of diffusion tensor tractography (DTT), which was reconstructed from diffusion tensor imaging (DTI), has enabled three-dimensional reconstruction and estimation of various neural tracts in the live human brain. ${ }^{3}$ Many studies using DTT have reported on injury of neural tracts in patients with traumatic brain injury. ${ }^{4-7}$ These neural tracts

Received Dec 9, 2021 Revised Dec 20, 2021

Accepted Dec 20, 2021

Corresponding author Jeong Pyo Seo

E-mail raphael0905@hanmail.net include the corticospinal tract, arcuate fasciculus, corticoreticulospinal tract, Papez circuit, spinothalamic tract, etc. ${ }^{4-7}$ However, little is known about injury of the CFT from the secondary motor area, which can be accompanied by bradykinesia, as an important clinical feature of limb-kinetic apraxia (LKA).

In this study, we report on a patient who developed mild bradykinesia due to injury of the CFT from the secondary motor area following direct head trauma, which was demonstrated on DTT.

\section{CASE REPORT}

A 58-year-old male patient underwent conservative management for subarachnoid hemorrhages caused by direct head trauma resulting from a fall from six-meter height at the department of neurosurgery of a local hospital. The patient lost consciousness for approximately three days and expe-

Copylight (C2021 The Korean Society of Physical Therapy

This is an Open Access article distribute under the terms of the Creative Commons Attribution Non-commercial License (https:// creativecommons.org/license/by-nc/4.o.) which permits unrestricted non-commercial use, distribution, and reproduction in any medium, provided the original work is properly cited. 
rienced post-traumatic amnesia continuously after regaining consciousness. His Glasgow Coma Scale score was 3. He developed mildly slow movements following the head trauma and visited the rehabilitation department of a university hospital at ten weeks after the fall. The patient exhibited mild bradykinesia during walking and arm movements with mild weakness in all four extremities $\left(\mathrm{G} / \mathrm{G}^{-}\right)$, and we observed shuffling gait and mutilated pattern of execution of arm movements. No specific lesion was observed on brain MRI (T1-weighted, T2-weighted, and Fluid attenuated inversion recovery [FLAIR] images) that was taken at ten weeks after onset (Figure 1A). One normal control subject with no history of neurologic disease was enrolled for comparison with the patient in terms of morphological characteristic of neural tract. The subjects provided writ-

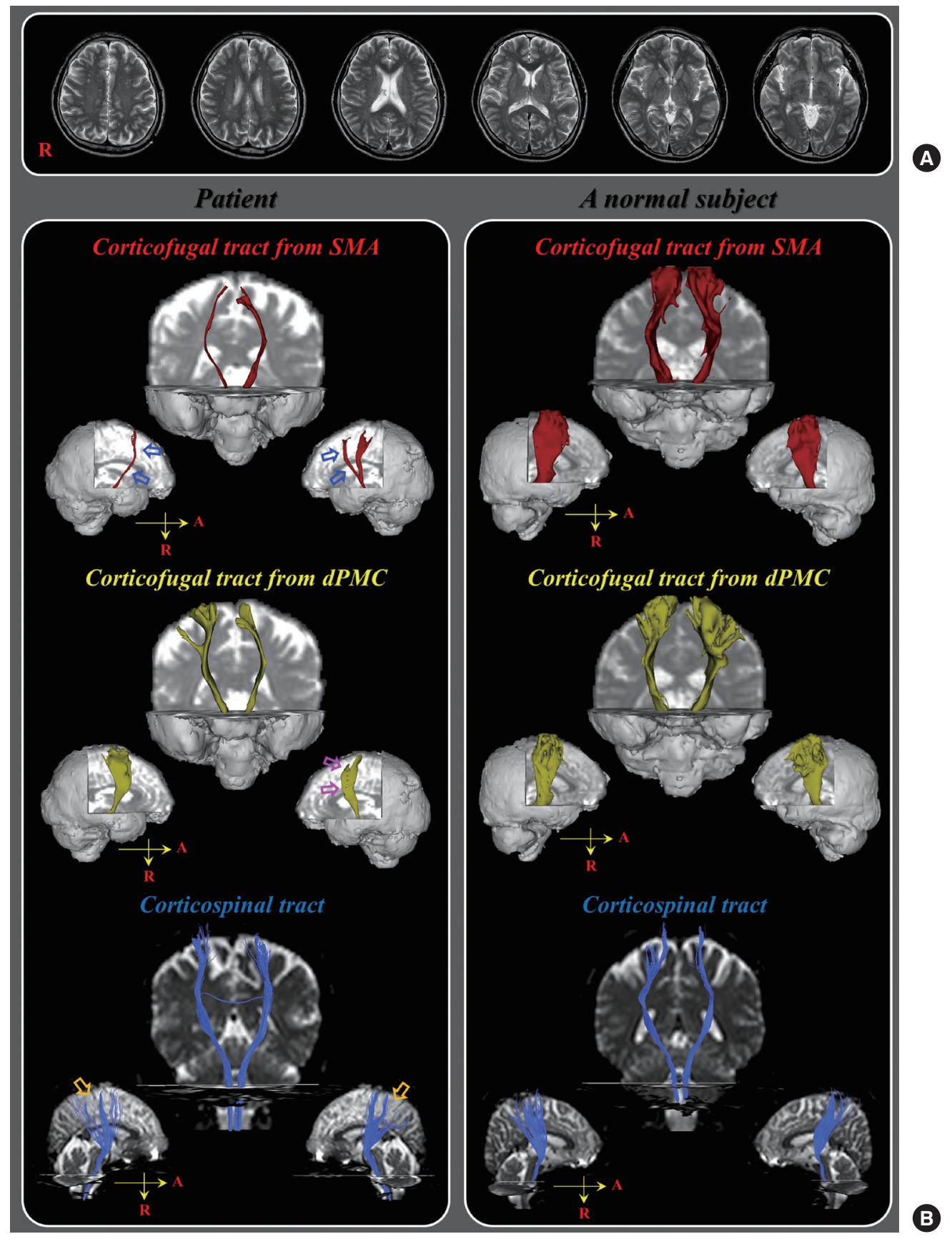

Figure 1. (A) T2-weighted brain MR images at 10 weeks after onset show no abnormality.(B) Results of 10-week diffusion tensor tractography. Narrowing of the right supplementary motor area-corticofugal tract (blue arrows) and partial tearing of the left supplementary motor area-corticofugal tract (purple arrows) and dorsal premotor cortex-corticofugal tract (purple arrows), and both corticospinal tract (orange arrows) at the subcortical white matter compared with those of a normal subject (56-year old male) are observed. 
ten informed consent prior to conduct of the study and the study protocol was approved by the institutional review board of a university hospital.

Diffusion tensor imaging (DTI) data was acquired at ten weeks after onset using a 6-channel head coil on a 1.5 T Philips Gyroscan Intera (Philips, Ltd, Best, The Netherlands) with single-shot echo-planar imaging. 70 contiguous slices (acquisition matrix: 96×96, reconstructed to matrix: 192 $\times 192$, field of view: $240 \times 240 \mathrm{~mm}^{2}$, TR: 10,398 ms, TE: $72 \mathrm{~ms}$, parallel imaging reduction factor: 2, echo-planar imaging factor: 59 and $b: 1,000$ $\mathrm{sec} / \mathrm{mm}^{2}$, NEX: 1 , and a slice thickness: $2.5 \mathrm{~mm}$ ) were acquired parallel to the anterior commissure-posterior commissure line. To analyze the corticospinal tract (CST), fiber tracking was performed using the fiber assignment continuous tracking algorithm implemented within the DTI task card software (Philips Extended MR Work Space 2.6.). The seed region of interest (ROI) was placed on the lower pons, and the target ROI was placed on the upper pons on the color map. Termination criteria used for fiber tracking were $\mathrm{FA}<0.15$, angle $<27^{\circ} .{ }^{8}$ For analysis of the CFTs from the dorsal premotor cortex (dPMC-CFT) and supplementary motor area (SMA-CFT), the Oxford Centre for Functional Magnetic Resonance Imaging of the Brain (FMRIB) Software Library (FSL; www.fmrib.ox.ac.uk/ fsl) was used for analysis of DTI data. For CFTs, the seed ROI was placed on the cerebral peduncle of the midbrain. The target ROI was defined as the dPMC and SMA area. ${ }^{8}$ Out of 5,000 samples generated from each seed voxel, results for each contact were visualized threshold and weightings of tract probability at a minimum of 2 streamlines through each voxel for analysis. On ten-week DTT, narrowing (decrement of track volum) of the right SMA-CFT, and partial tearing (rupture of part of the neural tract) of the left SMA-CFT, left dPMC-CFT and both CSTs at the subcortical white matter were observed (Figure 1B).

\section{DISCUSSION}

We found abnormalities in both CSTs from the primary motor cortex (partial tearing at the subcortical white matter and narrowing), both SMA-CFTs (narrowing and partial tearing) and left dPMC-CFT. We hypothesize that mild weakness in all four extremities was ascribed to partial tearing of both CSTs and mild bradykinesia to the injury of both SMACFTs and left dPMC-CFT.9 Injuries of both SMA-CFTs, left dPMC-CFT, and both CSTs indicated traumatic axonal injury, because the conventional brain MRI showed no abnormality. ${ }^{10,11}$ The CFTs from the secondary motor area (dPMC and SMA) are involved in motor planning, and in- jury of the CFTs from the secondary motor area is usually accompanied by LKA. ${ }^{2}$ Our patient showed mild bradykinesia, a typical mild symptom of LKA from the onset of direct head trauma. ${ }^{2}$

In the current study, using DTT, we demonstrated an association of bradykinesia with injured CFTs from the secondary motor area in patients with traumatic brain injury (TBI). We believe our findings suggest the necessity of assessment of the CFTs from the secondary motor area for patients with unexplained bradykinesia following TBI. Several DTT studies have reported on injury of the CFTs from the secondary motor area in the human brain. ${ }^{8,10,11}$ By contrast, to the best of our knowledge, this is the first study to demonstrate the relation of bradykinesia with injured CFTs from the secondary motor area in patients with TBI. However, because it is a case report, this study is limited. Conduct of further complementary studies involving larger numbers of cases is warranted.

\section{REFERENCES}

1. Newton JM, Ward NS, Parker GJ et al. Non-invasive mapping of corticofugal fibres from multiple motor areas-relevance to stroke recovery. Brain. 2006;129(7):1844-58.

2. Leiguarda RC, Marsden CD. Limb apraxias: higher-order disorders of sensorimotor integration. Brain. 2000;123(5):860-79.

3. Mori S, van Zijl PC. Fiber tracking: Principles and strategies - a technical review. NMR Biomed. 2002;15(7-8):468-80.

4. Jang SH, Lee AY, Shin SM. Injury of the arcuate fasciculus in the dominant hemisphere in patients with mild traumatic brain injury: a retrospective cross-sectional study. Medicine. 2016;95(9):e3007.

5. Jang SH, Kwon HG. Injury of the ascending reticular activating system in patients with fatigue and hypersomnia following mild traumatic brain injury: two case reports. Medicine. 2016;95(6):e2628.

6. Jang SH, Kim SY. Injury of the corticospinal tract in patients with mild traumatic brain injury: a diffusion tensor tractography study. J Neurotrauma. 2016;33(19):1790-5.

7. Yang DS, Kwon HG, Jang SH. Injury of the thalamocingulate tract in the papez circuit in patients with mild traumatic brain injury. Am J Phys Med Rehabil. 2016;95(3):e34-8.

8. Jang SH, Seo JP. Limb-kinetic apraxia due to injury of corticofugal tracts from secondary motor area in patients with corona radiata infarct. Acta Neurologica Belgica. 2016;116(4):467-72.

9. Jang SH, Kim SY. Injury of the corticospinal tract in patients with mild traumatic brain injury: a diffusion tensor tractography study. J Neurotrauma. 2016;33(19):1790-5.

10. Hong JH, Lee J, Cho YW et al. Limb apraxia in a patient with cerebral infarct: diffusion tensor tractography study. NeuroRehabilitation. 2012; 30(4):255-9.

11. Schulz R, Park CH, Boudrias MH et al. Assessing the integrity of corticospinal pathways from primary and secondary cortical motor areas after stroke. Stroke. 2012;43(8):2248-51. 